\title{
Distinct impacts of blood flow and temperature on cutaneous microvascular adaptation
}

\author{
Howard H. Carter ${ }^{1}$, Angela L. Spence ${ }^{1}$, Ceri L. Atkinson ${ }^{1}$, Christopher J.A. Pugh ${ }^{1}$, \\ N Timothy Cable ${ }^{2}$, Dick H.J. Thijssen ${ }^{2,3}$, Louise H. Naylor ${ }^{1}$, Daniel J. Green ${ }^{1,2}$ \\ ${ }^{1}$ School of Sport Science, Exercise and Health, The University Of Western \\ Australia, Crawley, Western Australia, 6009 \\ ${ }^{2}$ Research Institute for Sport and Exercise Science, Liverpool John Moores \\ University, Liverpool L3 2ET, United Kingdom \\ ${ }^{3}$ Department of Physiology, \\ Radboud University Nijmegen Medical Centre, The Netherlands

\section{Running Title:} \\ Skin adaptation to core temperature elevation
}

\section{Author Contributions}

D.J.G, H.H.C and D.H.J.T conceived and designed the experiments. H.H.C, A.L.S, C.L.A, C.J.A.P and L.H.N performed data collection. All authors were involved in data analysis, interpretation of the results and the writing of the manuscript. All authors helped revise the manuscript critically and have approved the final submitted version.

\section{Funding:}

Prof. Green is funded by the Australian Research Council (DP 130103793). The results of the present study do not constitute endorsement by the American College of Sports Medicine.

\section{Conflict of Interest:}

None of the authors have declared any conflict of interest

\section{Author for Correspondence:}

Howard Carter

School of Sport Science, Exercise and Health,

The University of Western Australia,

Crawley, Western Australia, 6009

Phone: +61 (8) 6488 2378, Fax: +61 (8) 64881039

Email: carteh01@student.uwa.edu.au 


\section{Abstract}

Aims: We performed two experiments to determine whether cutaneous microvascular adaptations in response to repeated core temperature elevation are mediated by increases in skin temperature, and/or, skin blood flow. Methods: Healthy subjects participated for 8weeks in thrice-weekly bouts of 30 mins lower limb heating $\left(40^{\circ} \mathrm{C}\right)$. In Study 1 , both forearms were "clamped" at basal skin temperature throughout each heating bout $(n=9)$. Study 2 involved identical lower limb heating, with the forearms under ambient conditions (unclamped, $\mathrm{n}=10$ ). In both studies, a cuff was inflated around one forearm during the heating bouts to assess the contribution of skin blood flow and temperature responses. We assessed forearm skin blood flow responses to both lower limb (systemic reflex) heating, and to local heating of the forearm skin, pre and post intervention. Results: Acutely, lower limb heating increased core temperature (Study 1: $+0.63 \pm 0.15^{\circ} \mathrm{C}$, Study 2: $+0.69 \pm 0.19^{\circ} \mathrm{C}$, $\mathrm{P}<0.001$ ) and forearm skin blood flow (Study 1: $10 \pm 3$ vs $125 \pm 44$, Study 2: $16 \pm 9$ vs $136 \pm 41$ $\mathrm{PU}, \mathrm{P}<0.001)$, with skin responses significantly attenuated in the cuffed forearm $(\mathrm{P}<0.01)$. Skin blood flow responses to local heating decreased in Study 1 (clamped forearms, week 0vs8: $1.46 \pm 0.52$ vs $0.99 \pm 0.44$ CVC, $\mathrm{P}<0.05$ ), whereas increases occurred in Study 2 (unclamped; week 0vs8: $1.89 \pm 0.57$ vs $2.27 \pm 0.52$ CVC, $\mathrm{P}<0.05$ ). Cuff placement abolished local adaptations in both studies. Conclusion: Our results indicate that repeated increases in skin blood flow and skin temperature result in increased skin flux responses to local heating, whereas repeated increases in skin blood flow in the absence of change in skin temperature induced the opposite response. Repeated increases in core temperature induce intrinsic microvascular changes, the nature of which are dependent upon both skin blood flow and skin temperature.

Keywords: Core temperature, local heating, skin blood flow, thermoregulation 


\section{Introduction}

Paragraph Number 1. A recent study demonstrated that 8 weeks of bilateral forearm heating resulted in increased skin blood flow (SkBF) responsiveness to a local heating protocol (5). No cutaneous microvascular adaptation was evident in this experiment when the increases in SkBF associated with repeated forearm heating were attenuated by unilateral inflation of a pneumatic cuff. It was concluded that increased blood flow, and the associated increase in shear stress, play a role in enhancing microvascular vasodilator function in humans. However, this experiment did not exclude a role for heat delivery, attendant to cutaneous hyperemia. Both blood flow and temperature are known physiological stimuli for vascular adaptation $(5,15,21)$ in humans.

Paragraph Number 2. In the present study, we therefore utilised two novel experimental models to dissect the independent effects of repeated increases in SkBF (and microvascular shear stress), versus increases in skin temperature, on cutaneous microvascular adaptation in humans. In the first study, subjects underwent 3 x weekly bouts of 30 mins of lower limb heating $\left(40^{\circ} \mathrm{C}\right)$ in a custom-designed immersion bath which isolated heating to the lower limbs. Throughout each of these heating bouts, a pneumatic cuff was positioned and inflated around one forearm to unilaterally manipulate SkBF in the upper limbs, which allowed for a within-subject bilateral assessment of the role of blood flow (and shear) in microvascular adaptation to repeated increases in core temperature (Tc). To further isolate the impact of changes in SkBF from those associated with blood flow-mediated increases in skin temperature, both forearms were 'clamped' at $30^{\circ} \mathrm{C}$ via immersion in thermostaticallycontrolled baths throughout each bout of lower limb heating in study 1 . A subsequent experiment (Study 2) was identical in design (8 weeks, 3 x weekly, lower limb heating, unilateral cuff inflation), with the important difference that both forearms were in ambient 
conditions during the heating bouts; that is, skin temperatures were 'unclamped' and therefore allowed to increase with the rise in SkBF that accompanied Tc elevation. Collectively these experiments, involving repeated Tc elevation, allowed us to isolate the impact of increases in SkBF (and hence microvascular shear stress) per se, from the effects of repeated increases in skin temperature. We hypothesised that repeated increases in shear stress would enhance the intrinsic capacity of the skin to vasodilate, and that the combination of increased SkBF and elevated local skin temperature would further enhance this localised vascular adaptation.

\section{Materials and Methods}

\section{Ethical Approval}

Paragraph Number 3. This study complied with the Declaration of Helsinki and the Human Research Ethics Committee of the University of Western Australia approved the experimental protocol. All subjects provided written, informed consent before participating in the study.

\section{Subject Characteristics}

Paragraph Number 4. Nine young, healthy recreationally active ( $\leq 2 \mathrm{hrs}$ of physical activity per week) males were recruited for Study 1 and 10 were recruited for Study 2 (Table 1). Subjects had no history of cardiovascular, musculoskeletal or metabolic disease, did not smoke or take medication. Women were excluded from this study due to the well-established effects of oestrogen on hemodynamic and vascular responses. All subjects commenced the intervention between the months of May-July and were therefore exposed to similar natural environments prior to and throughout the interventions. 


\section{Study Designs}

Study 1: Repeated lower limb heating, unilateral cuff inflation to manipulate forearm SkBF, forearm temperatures clamped $\left(30^{\circ} \mathrm{C}\right)$

Paragraph Number 5. All subjects underwent baseline assessments and were then required to attend the laboratory 3 times per week, for 8 weeks. During each of these sessions, subjects were seated in a custom-designed inflatable recovery bath (IC-iBody; iCoolsport, Queensland, Australia) and immersed up to their waist in warm water $\left(40^{\circ} \mathrm{C}\right)$ for a period of 30 minutes. The water bath temperature was maintained and continuously circulated via a thermostatic heating pump (IC-Heat; iCoolsport, Queensland, Australia). A thick plastic sheet was placed over this bath to isolate heating to the legs, leaving the upper body unaffected by the heating stimulus.

Paragraph Number 6. To eliminate the effect of lower limb heating and Tc-induced reflex increases in SkBF on forearm temperature, each forearm was immersed up to the elbows in thermostatically controlled euthermic water throughout each of the 30 min lower limb heating bouts. Pilot studies indicated that resting forearm skin temperatures averaged $\sim 30^{\circ} \mathrm{C}$ in our laboratory, therefore these water baths were set and maintained at this basal temperature. Finally, a pneumatic cuff was positioned and inflated to $80 \mathrm{mmHg}$ around one forearm throughout each of the 30 min heating periods, to attenuate the increase in SkBF induced by elevation in Tc in this limb. In this way, we controlled for the impact of skin temperature during core heating whilst manipulating SkBF bilaterally. 
Study 2: Repeated lower limb heating, unilateral cuff inflation to manipulate forearm SkBF, forearm temperatures unclamped

Paragraph Number 7. This experiment was identical to the one above, with the exception that the forearms were not placed in temperature baths during the repeated episodes of lower limb heating. By comparing the results of this experiment to that above, we were able to address the impact of repeated skin temperature elevation on cutaneous microvascular adaptation in response to core heating.

\section{Experimental Outcomes}

Acute impact of leg heating on forearm skin blood flow responses at study entry

Paragraph Number 8. At study entry, to assess the efficacy of manipulation of our independent variable, SkBF's were determined using 7 Doppler array laser probes (Model 413; Periflux 5001 System, Perimed AB, Sweden) in both experimental studies described above. That is, skin perfusion was assessed across a $30 \mathrm{~min}$ period during isolated lower limb heating $\left(40^{\circ} \mathrm{C}\right)$, under circumstances where both forearms were immersed at $30^{\circ} \mathrm{C}$ (Study 1, Figure 1 A-B) and both forearms were under ambient conditions (Study 2, Figure 1 C-D). The impact of forearm cuff placement during both studies was also assessed. Skin blood flow measures were collected simultaneously in both arms, effectively eliminating any impact of systemic hemodynamics. The data therefore are presented in perfusion units (PU).

Impact of repeated lower limb heating on the relationship between core temperature and forearm skin perfusion responses

Paragraph Number 9. To assess the impact of repeated lower limb heating on adaptations in the relationship between Tc elevation and skin perfusion, a sub-group of 6 subjects (23.3 \pm $1.8 \mathrm{yrs})$ in study 1 and 8 subjects (25.8 \pm 3.1 yrs) in study 2 had measures collected 
throughout the heating protocols described above. Laser Doppler (Model 413; Periflux 5001 System, Perimed AB, Sweden) was used to record changes in SkBF throughout the 30 min leg immersions. Core temperature was measured from rectal temperature probes throughout immersion (RET-1, Physitemp Instruments, NJ, USA). In this way it was possible to assess the impact of repeated episodic elevation in Tc on adaptations in forearm skin perfusion during an acute bout of lower limb heating undertaken pre- and post-intervention.

Impact of repeated lower limb heating on skin blood flow responses to localised heating Paragraph Number 10. At weeks 0 and 8, all subjects attended an experimental laboratory session having fasted for a minimum of 8 hours and abstained from alcohol, caffeine and vigorous exercise for at least 24 hours. These studies were conducted in a quiet, euthermic environment with subjects at rest and seated comfortably. The assessments were performed at the same time of day. They did not involve lower limb heating or cuffing or any intervention other than localised heating of the skin using heater discs, as described below.

Paragraph Number 11. Laser Doppler probe sites on each forearm were shaved and cleaned 24 hours prior to the laboratory attendance. Photographs were taken of the sites and measurements made from bony anthropometric landmarks at baseline so that similar placement sites were selected on each forearm for repeated measures at 8 weeks. Local heater discs (Perimed 455, Stockholm, Sweden) were attached to the forearms using double-sided adhesive rings. The 7 laser Doppler array probes (Model 413; Periflux 5001 System, Perimed AB, Sweden) were then fitted into the middle of the localised heating discs. Room temperature was controlled and recorded throughout all assessments. 
Paragraph Number 12. Once instrumented, the heater discs were increased to $33^{\circ} \mathrm{C}$ and remained at this temperature for a 20 min baseline period. The heater discs were then increased in increments of $0.5^{\circ} \mathrm{C}$ every 5 minutes until $42^{\circ} \mathrm{C}$ was reached, so as to minimise any impact of axon reflexes (13) which are less NO dependent than the protocol adopted in this study (1). Finally, the heater discs remained at $42^{\circ} \mathrm{C}$ for a further 30 mins. A previous paper established that SkBF in response to this gradual heating protocol is largely NOmediated $(1,14)$. Blood pressure was recorded every 5 mins at the ankle using a Dinamap automated monitor and later corrected for the hydrostatic column (6) and used to calculate cutaneous vascular conductance (CVC). All laser Doppler, room and core temperature measurements were relayed and recorded in real time onto a laptop using the software program LabChart 7 (ADinstruments, Sydney, Australia).

\section{Data Analysis}

\section{Laser Doppler Data}

Paragraph Number 13. Skin PU data from the cuffed and uncuffed arms were averaged over a stable $30 \mathrm{sec}$ period at the end of every 5 min interval to assess SkBF. Calibration of the probes were undertaken before the experiments using two generic points, 0 and $250 \mathrm{PU}$, in accordance with calibration guidelines using a zeroing disk and motility standard (Periflux System, Perimed AB, Sweden). Measurements in PU were converted to CVC which was calculated as PU / Dinamap mean arterial pressure (MAP). All core and room temperature readings were averaged at the end of each 5 min interval in ${ }^{\circ} \mathrm{C}$.

\section{Statistics}

Paragraph Number 14. Skin blood flow PU and CVC outcome data were compared withinsubjects, across 2 study time points (0 and 8 weeks) using 2-factor ANOVA with planned 
comparisons performed on four temperature points; $34^{\circ} \mathrm{C}, 40-42^{\circ} \mathrm{C}$ and $42^{\circ} \mathrm{C}+30$ mins. Post hoc $t$-tests were performed where significance was detected at $\mathrm{P}<0.05$, using LSD tests to correct for multiple comparisons.

\section{Results}

Acute impact of lower limb heating on forearm skin blood flow responses at study entry

Study 1: Repeated lower limb heating, unilateral cuff inflation to manipulate forearm SkBF, forearm temperatures clamped $\left(30^{\circ} \mathrm{C}\right)$

Paragraph Number 15. Despite being bathed at a constant skin temperature of $30^{\circ} \mathrm{C}$, forearm SkBF increased significantly in both the uncuffed and cuffed forearms during 30 mins of lower limb heating at $40^{\circ} \mathrm{C}(\mathrm{P}<0.001$ and $\mathrm{P}<0.001$, respectively; Figure $1 \mathrm{~A})$. However, inflation of the cuff around one forearm significantly attenuated the increase in SkBF compared to the uncuffed arm $(\mathrm{P}<0.01)$. Skin temperatures were maintained in both arms at $30^{\circ} \mathrm{C}$ throughout the heating session (Figure 1B).

Study 2: Repeated lower limb heating, unilateral cuff inflation to manipulate forearm SkBF, forearm temperatures unclamped

Paragraph Number 16. Resting SkBF was similar between forearms $(\mathrm{P}=0.26)$. Skin blood flow increased in both the uncuffed and cuffed arms during 30 mins of lower limb heating $(\mathrm{P}<0.001$ and $\mathrm{P}<0.001$, respectively; Figure $1 \mathrm{C})$. Inflation of the cuff significantly reduced the increase in SkBF in the cuffed arm compared to the uncuffed $(\mathrm{P}<0.001)$, with differences at all time points $(\mathrm{P}<0.05)$ (Figure $1 \mathrm{C})$. Similarly, skin temperatures in the uncuffed arm significantly increased across the 30 min lower limb heating period $(\mathrm{P}<0.001)$. However, in 
the contralateral limb, cuff inflation abolished increases in skin temperature throughout the heating bout (Figure 1D).

Comparison of skin blood flow and temperature between the studies

Paragraph Number 17. No difference was evident between SkBF responses in the uncuffed arms throughout the lower limb heating bouts between the studies $(\mathrm{P}=0.19$, Figure $1 \mathrm{~A}, \mathrm{C}$, closed symbols). A significant difference in uncuffed limb skin temperature responses was evident between the studies $(\mathrm{P}<0.01)$, indicative of the impact of forearm water immersion at $30^{\circ} \mathrm{C}$.

Paragraph Number 18. The data above therefore indicate that, whilst SkBF increased in the uncuffed arm in both experiments, skin temperature was effectively controlled in study 1 . Similarly, cuff placement effectively attenuated SkBF responses between the limbs. These experimental manipulations therefore allow us to distinguish between the impacts of repeated elevations in SkBF (and microvascular shear stress) in the presence and absence of affiliated increases in skin temperature.

Impact of repeated lower limb heating on the relationship between core temperature and forearm skin perfusion responses

Study 1: Repeated lower limb heating, forearm temperatures clamped $\left(30^{\circ} \mathrm{C}\right)$

Paragraph Number 19. During the first and last bout of 30 min heating (ie week 0 and 8), the forearms were in ambient air for both experiments so that the impact of repeated core heating on the relationship between Tc and SkBF could be assessed. 
Paragraph Number 20. Forearm perfusion did not change across the 8 weeks in response to lower limb heating ( $\mathrm{P}=0.73$; Figure $2 \mathrm{~A})$, whereas $\mathrm{Tc}$ responses to lower limb heating decreased after 8 weeks $(\mathrm{P}<0.05$; Figure $2 \mathrm{~B})$. When change in SkBF was plotted against change in Tc across the 30 min session at week 0 and 8, a higher SkBF was apparent for a given change in Tc (Figure 3A).

Study 2: Repeated lower limb heating, forearm temperatures unclamped

Paragraph Number 21. In keeping with the data above, forearm perfusion did not significantly change across the 8 weeks in response to lower limb heating in this experiment $(\mathrm{P}=0.30$; Figure 2C), whereas resting Tc at week 8 was significantly lower compared to week 0 (37.2 \pm 0.3 vs $36.9 \pm 0.2, \mathrm{P}<0.001)$. Similarly, Tc was significantly lower during lower limb heating at week 8 compared to week $0(\mathrm{P}<0.001)$ with differences at every 5 min interval $(\mathrm{P}<0.05)$ across the 30 mins (Figure 2D). When change in SkBF was plotted against change in Tc across the 30 min session at week 0 and 8, a higher SkBF was evident for a given change in Tc (Figure 3B). No differences were evident between MAP responses during the lower limb heating bouts at weeks 0 and 8 (Weeks 0 vs $8 ; 97 \pm 8$ vs $96 \pm 6,87 \pm 7$ vs $89 \pm 6,86 \pm 7$ vs $87 \pm 5$ and $86 \pm 9$ vs $87 \pm 7, P=0.88)$.

Impact of repeated lower limb heating on skin blood flow responses to a standardised local heating stimulus

Study 1: Repeated lower limb heating, unilateral cuff inflation to manipulate forearm SkBF, forearm temperatures clamped $\left(30^{\circ} \mathrm{C}\right)$

Paragraph Number 22. Mean CVC values at $33^{\circ} \mathrm{C}$ were similar between weeks 0 and 8 in the cuffed arm $(0.29 \pm 0.10$ vs $0.29 \pm 0.11, \mathrm{P}=0.87)$. Additionally, there was no significant 
difference between weeks 0 and 8 in responses to incremental heating in the cuffed arm $(\mathrm{P}=0.72$; Figure 4A).

Paragraph Number 23. In the uncuffed arm, mean $\mathrm{CVC}$ values at $33^{\circ} \mathrm{C}$ remained similar between weeks 0 and $8(0.21 \pm 0.09$ vs $0.17 \pm 0.08, \mathrm{P}=0.16)$. However, a decrease in SkBF responsiveness to incremental heating throughout the local heating protocol was evident at week 8 compared to baseline ( $\mathrm{P}=0.05$; Figure 4B).

Study 2: Repeated lower limb heating, unilateral cuff inflation to manipulate forearm SkBF, forearm temperatures unclamped

Paragraph Number 24. Mean CVC values at $33^{\circ} \mathrm{C}$ were similar between weeks 0 and 8 in the cuffed arm (0.22 \pm 0.06 vs $0.29 \pm 0.09)$ and there was no significant difference in the response to incremental heating between weeks 0 and 8 in the cuffed arm $(P=0.69$; Figure 4C).

Paragraph Number 25. In the uncuffed arm, mean $\mathrm{CVC}$ values at $33^{\circ} \mathrm{C}$ remained similar across weeks 0 and $8(0.20 \pm 0.07$ vs $0.23 \pm 0.11)$. In contrast to the results from study 1 , above, SkBF responses to incremental heating between weeks 0 and 8 increased $(\mathrm{P}<0.05$; Figure 4D).

Change in response to local heating: Direct comparison of the effect of skin temperature manipulation

Paragraph Number 26. A significant difference was apparent between change in CVC (baseline to week 8) at temperature points $40^{\circ} \mathrm{C}, 41.5^{\circ} \mathrm{C}$ and $42^{\circ} \mathrm{C}-30$ mins between Studies 1 and 2 (2-way between-subjects ANOVA, all post hoc $\mathrm{P}<0.05$, Figure 5). 


\section{Discussion}

Paragraph Number 27. In the present study, 8 weeks of repeated thermoregulatory reflexmediated increase in forearm SkBF induced divergent cutaneous microvascular adaptations to a local heating stimulus, dependent upon whether skin temperatures were elevated or not during the lower limb heating bouts. When skin temperatures were prevented from rising across the 8 week intervention period (Study 1), post-intervention localised heating elicited decreases in microvascular perfusion, whereas repeated elevation in both SkBF and temperature (Study 2) elicited increased perfusion responses to our standardised local heating test. Finally, when both SkBF and temperature were attenuated, using cuff placement, no adaptations were apparent in either study. Taken together, these data suggest that intrinsic cutaneous microvascular adaptation is dependent upon repetitive episodic increases in blood flow, and that skin temperature modifies the direction of this response.

Paragraph Number 28. A recent study involving thrice weekly bouts of bilateral forearm heating $\left(42^{\circ} \mathrm{C}\right)$ for 8 weeks elicited an increase in SkBF responses to local heating in the uncuffed, but not the cuffed, forearm (5). This study suggested that SkBF and/or skin temperature were key stimuli for cutaneous microvascular adaptation, but it did not allow us to distinguish between these stimuli. This previous study involved localised forearm heating, and did not induce Tc elevation or reflex thermoregulatory changes. We therefore devised the current experiments to assess the relative impact of repeated increases in blood flow (shear) and/or skin temperature on cutaneous microvascular adaptations in response to thermoregulatory-reflex mediated increases in forearm SkBF. In these studies, we heated the legs of subjects whilst clamping skin temperatures (study 1) in the presence of rising SkBF's, 
whereas in study 2 forearm skin temperatures were not clamped and were allowed to rise with flow (study 2). Interestingly, and in contrast to our expectations, we observed distinct cutaneous microvascular adaptations in these experiments. Microvascular perfusion in response to a standardised local heating test increased following the latter intervention, but decreased in the former.

Paragraph Number 29. The decrease in skin perfusion during localised heating that we observed in study 1 may conceivably relate to functional or structural microvascular adaptation. It is well established that SkBF is regulated by both neural and local mechanisms (9). For example, rapid local heating of the skin induces a transient peak in SkBF mediated by axon reflexes, a response followed by prolonged vasodilation mediated by the local release of NO (13). The gradual local heating test we utilised in the present study is considered to be largely NO mediated (1). In contrast, whole body heating elicits central thermoregulatory reflexes which increase cutaneous vasodilator outflow via sympathetic cholinergic nerves (9). There are several proposed mechanisms by which vasodilation is induced by this neural drive, including the co-transmitter concept whereby sweating and vasodilation are stimulated by the release of ACh and an as yet undetermined vasodilator substance $(9,22)$. Recently, Wong reported that inhibition of sensory nerves and NO production reduced the reflex vasodilator response to whole body heating by $\sim 80 \%$ and that NO is obligatory for full expression of reflex-mediated vasodilation in the skin (22). It was furthermore suggested that skin temperature-mediated activation of sensory nerves may play a distinct role in reflex vasodilation, compared to that associated with endothelium-dependent mechanisms. The present study adds to these important observations relating to the acute impacts of body heating, by addressing the chronic effects of repeated Tc elevation mediated by skin perfusion versus hyperemia associated with increased skin temperature. Future 
studies, similar to those described above (22) involving blockade of specific transmitters will provide further mechanistic insight into the distinct impacts of repeated episodic changes in skin perfusion and temperature in vivo.

Paragraph Number 30. It is possible that, in the absence of changes in localised forearm skin temperature during our lower limb bath exposures, one or more of the many intrinsic vasodilators known to contribute to SkBF such as NO, EDHF, or prostacyclin may have been down-regulated. We believe this explanation is somewhat unlikely, as repeated increases in blood flow and shear per se have not previously been associated with decreased expression of vasodilator substances in other arteries (7). Nonetheless, our study suggests that repeated Tc elevation, in the absence of changes in skin temperature or extrinsic skin heating, does not up-regulate reflex-mediated vasodilation. An alternate explanation for our finding in study 1 invokes microvascular structural change. Although speculative, an increase in skin capillarisation following repeated Tc heating and blood flow (shear) stimulation might account for the decreases in skin flux we observed in response to local heating. Consequential prolongation in red cell transit time would enhance heat dissipation in the face of elevated Tc. By analogy, a similar prolongation in transit time due to increased capillarity has been proposed in skeletal muscle following exercise training (10). Unfortunately, we did not directly assess structural microvascular changes in the present study and future studies will therefore be required to elicit maximal CVC (i.e. local heating to $44^{\circ} \mathrm{C}$ or delivery of vasoactive substances via microdialysis) to confirm changes in skin capillarity. Whilst we are not aware of any previous direct evidence that skin capillarisation increases in response to either exercise training or repeated passive elevation in Tc, this possibility is consistent with our findings and previously reported changes in skeletal muscle (10) and worthy of future study. 
Paragraph Number 31. A possible explanation for the increase in SkBF response to local heating that we observed in study 2, and also in our previous forearm heating experiment (5), may relate to the bioavailability of vasodilators in response to the significant increases in skin temperature. It is known that increases in temperature enhance the expression of heat shock proteins (HSPs), and there is a known association between HSP90 and eNOS $(4,18)$. Indeed, Shastry et al. reported that inhibition of HSP90 (by geldanamycin) reduced SkBF in response to local heating and acetylcholine, highlighting the essential role of HSP90 in full eNOS activation (19). In this context, it is possible to rationalise the current findings as reflective of heating-induced expression of HSP90 (or other HSPs) and subsequent activation of eNOS, thereby enhancing functional vasodilator capacity. Such a cascade may not be apparent in study 1 , because of the lack of increase in skin temperature during the lower limb heating bouts. We also cannot discount the possibility that clamping skin temperatures in Study 1 to $30^{\circ} \mathrm{C}$ altered the association between eNOS and HSP's, or other vasodilators, in such a way that SkBF responses to local heating were reduced due to functional down-regulation. However, it seems unlikely that preventing activation of vasodilator pathways by maintaining skin temperature at euthermic levels would actively down-regulate microvascular function. For this reason, and because repeated shear stress has previously been associated with structural arterial remodelling $(2,11)$, we suggest that increased capillarsation may have resulted from the repeated episodic hyperemia we induced in study 1.

Paragraph Number 32. Apart from the adaptations referred to above, which pertain to vasodilation in response to localised heating, we also measured SkBF and Tc changes during lower limb heating bouts, before and after the intervention period. It is generally accepted that acclimation results in increases in SkBF at an earlier Tc, and to a higher plateau level 
(20). Classic human integrative physiology studies furthermore suggest that whole-body heat exposure during brief periods of exercise training (referred to by some as "acclimation”), exaggerates this response (16). A recent review (20) and paper (8) attributed these effects on the Tc/SkBF relationship to changes in blood volume, although the latter study occurred across a brief timeframe and there is some evidence that blood volume changes are timedependent (17). The relative increase in SkBF for a given Tc that we observed in both current experiments (Figures 2 and 3) is consistent with that previously observed $(3,20)$ and strongly implies central blood volume expansion. However, a recent and carefully performed study by Lorenzo and Minson demonstrated that heat acclimation involving 10 days of combined exercise and $40^{\circ} \mathrm{C}$ whole body heat exposure induces localised SkBF adaptations in response to acetylcholine administration (12). Our study design was different to that of Lorenzo and Minson and sought to answer distinct questions, but if the mechanism for our observed change in Tc/SkBF relationship during lower limb heating was peripheral, rather than central (e.g. blood volume related), then it must relate to episodic increases in skin perfusion and not changes in skin temperature as the Tc/SkBF change was observed in both of our experiments.

Paragraph Number 33. One potential limitation of the present study relates to the placement of the laser Doppler probes before and after the intervention. It is possible that differential placement may have affected the changes we observed. However we believe our findings are robust and internally consistent for the several reasons. Each subject's initial baseline probe placement was recorded using distances from anatomical landmarks and a photograph was taken to ensure follow-up placement was as close as possible to the baseline site. Furthermore, the study design was within-subjects and between arms and truly random effects should have similarly impacted both limbs. Finally, the findings of our study were 
very consistent within conditions: of the 9 subjects in study 1, 8 demonstrated decreases in response to local heating, whereas 8 of 10 subjects increased in study 2.

Paragraph Number 34. In summary, this experiment suggests that repetitive increases in skin microvascular hyperemia, when associated with increased skin temperature, induces distinct adaptations to those associated with repeated increases in blood flow per se. Whilst episodic increases in SkBF induce microvascular changes consistent with prolonged red blood cell transit time, increases in skin temperature may be required to manifest functional adaptation and enhanced red cell flux. Interventions that repeatedly increase Tc and, consequently, both skin blood flow and skin temperature, likely induce central adaptations in blood volume as well as intrinsic microvascular changes which combine to enhance thermoregulation. 


\section{Acknowledgements}

Prof. Green is funded by the Australian Research Council (DP 130103793). We would like to sincerely thank Mr Craig Cook and iCoolsport for providing the custom designed recovery baths.

\section{Conflict of Interest}

None of the authors have declared any conflict of interest.

The results of the present study do not constitute endorsement by the American College of Sports Medicine. 


\section{References}

1. Black MA, Green DJ, Cable NT. Exercise training prevents age-related decline in nitric oxide (NO)-mediated vasodilator function in human microvessels. J. Physiol. (Lond.). 2008;586:3511-24.

2. Brown MD. Exercise and coronary vascular remodelling in the healthy heart. Exp. Physiol. 2003;88:645-58.

3. Fox RH, Goldsmith R, Kidd DJ, Lewis HE. Blood flow and other thermoregulatory changes with acclimatization to heat. J. Physiol. (Lond.). 1963;166:548-62.

4. Garcia-Cardeña G, Fan R, Shah V et al. Dynamic activation of endothelial nitric oxide synthase by Hsp90. Nature. 1998;392:821-4.

5. Green DJ, Carter HH, Fitzsimons MG, Cable NT, Thijssen DHJ, Naylor LH. Obligatory role of hyperaemia and shear stress in microvascular adaptation to repeated heating in humans. J. Physiol. (Lond). 2010;588:1571-7.

6. Groothuis JT, Poelkens F, Wouters CW, Kooijman M, Hopman MTE. Leg intravenous pressure during head-up tilt. J Appl Physiol. 2008;105(3):811-5.

7. Hambrecht R, Adams V, Erbs S et al. Regular Physical Activity Improves Endothelial Function in Patients With Coronary Artery Disease by Increasing Phosphorylation of Endothelial Nitric Oxide Synthase. Circulation. 2003;107(25):3152-8.

8. Ikegawa S, Kamijo Y-i, Okazaki K, Masuki S, Okada Y, Nose H. Effects of hypohydration on thermoregulation during exercise before and after 5-day aerobic training in a warm environment in young men. J Appl Physiol. 2011;110(4):972-80.

9. Kellogg DL. In vivo mechanisms of cutaneous vasodilation and vasoconstriction in humans during thermoregulatory challenges. J Appl Physiol. 2006;100(5):1709-18. 
10. Krustrup P, Hellsten Y, Bangsbo J. Intense interval training enhances human skeletal muscle oxygen uptake in the initial phase of dynamic exercise at high but not at low intensities. J Physiol. 2004;559(1):335-45.

11. Langille BL, O'Donnell F. Reductions in arterial diameter produced by chronic decreases in blood flow are endothelium-dependent. Nature. 1986;231:405-7.

12. Lorenzo S, Minson CT. Heat acclimation improves cutaneous vascular function and sweating in trained cyclists. J Appl Physiol. 2010;109(6):1736-43.

13. Minson CT, Berry LT, Joyner MJ. Nitric oxide and neurally mediated regulation of skin blood flow during local heating. J Appl Physiol. 2001;91(4):1619-26.

14. Minson CT, Holowatz LA, Wong BJ, Kenney WL, Wilkins BW. Decreased nitric oxide- and axon reflex-mediated cutaneous vasodilation with age during local heating. J. Appl. Physiol. 2002;93(5):1644-9.

15. Naylor LH, Carter HH, FitzSimons MG, Cable NT, Thijssen DHJ, Green DJ. Repeated increases in blood flow, independent of exercise, enhance conduit artery vasodilator function in humans. Am. J. Physiol.- Heart. C. 2011;300:664-9.

16. Roberts MF, Wenger CB, Stolwijk JA, Nadel ER. Skin blood flow and sweating changes following exercise training and heat acclimation. $J$ Appl Physiol. 1977;43(1):133-7.

17. Sawka MN, Convertino VA, Eichner ER, Schneider SM, Young AJ. Blood volume: Importance and adaptations to exercise training, environmental stresses, and trauma/sickness. Med. Sci. Sports Exerc. 2000;32:332-48.

18. Shah V, Wiest R, Garcia-Cardena G, Cadelina G, Groszmann RJ, Sessa WC. Hsp90 regulation of endothelial nitric oxide synthase contributes to vascular control in portal hypertension. Am J Physiol - Gastro L. 1999;277(2):G463-G8. 
Carter et al. MSSE Skin adaptation to core temperature elevation

19. Shastry S, Joyner MJ. Geldanamycin attenuates NO-mediated dilation in human skin. Am J Physiol - Heart C. 2002;282(1):H232-H6.

20. Simmons GH, Wong BJ, Holowatz LA, Kenney WL. Changes in the control of skin blood flow with exercise training: where do cutaneous vascular adaptations fit in? Experimental Physiology. 2011;96(9):822-8.

21. Tinken TM, Thijssen DHJ, Hopkins ND, Dawson EA, Cable NT, Green DJ. Shear stress mediates vascular adaptations to exercise training in humans. Hypertension. 2010;55:312-8.

22. Wong BJ. Sensory nerves and nitric oxide contribute to reflex cutaneous vasodilation in humans. Am J Physiol - Reg I. 2013;304(8):R651-R6. 


\section{Figure Legends}

Figure 1. Forearm skin blood flow (in perfusion units (PU)) (Study 1, A; Study 2, C) and temperature (Study 1, B; Study 2, D) in the cuffed (open squares) and uncuffed (closed squares) forearms at baseline and during 30 mins of lower limb heating (5 min intervals). "Significantly different at $\mathrm{P}<0.05$ from baseline. *Significantly different between the cuffed and uncuffed forearms at $\mathrm{P}<0.05$. SkBF- skin blood flow. Data are mean \pm SE.

Figure 2. Forearm skin blood flow (in perfusion units (PU)) (Study 1, A; Study 2, C) and core temperature (Study 1, B; Study 2, D) in response to 30 mins of lower limb heating at weeks 0 (open circles) and 8 (closed circles). ${ }^{\#}$ Significantly different at $\mathrm{P}<0.05$ from baseline. *Significantly different between weeks at $\mathrm{P}<0.05$. ${ }^{\dagger}$ Significantly different between weeks at $\mathrm{P}=0.05$. SkBF- skin blood flow. Data is mean \pm SE.

Figure 3. Change in forearm skin blood flow (in perfusion units (PU)) (y axis) versus change in core temperature (x axis) during 30 mins of lower limb heating (5 min intervals) at weeks 0 (open circles) and 8 (closed circles) in Study 1 (A) and Study 2 (B). SkBF- skin blood flow. Tc- core temperature. Data are derived from Figure 2.

Figure 4. Forearm skin blood flow (cutaneous vascular conductance (CVC)) during the entire gradual heating protocol in the cuffed (Study 1, A; Study 2, C) and uncuffed (Study 1, B; Study 2, D) arms at weeks 0 (open circles) and 8 (closed circles). LDF- laser Doppler flux. MAP- mean arterial pressure. Data is mean $\pm \mathrm{SE}$. 
Figure 5. Change in cutaneous vascular conductance (CVC) from week 0 to week 8 in the uncuffed arm in Study 1 (open columns) and Study 2 (closed columns). *Significantly different between studies at $\mathrm{P}<0.05$. Data is mean $\pm \mathrm{SE}$. 